\title{
Multi-objective hydropower purchase optimization method for inter-provincial power grids under time-sharing electricity price
}

\author{
Lu Peng ${ }^{1}, \mathrm{Li} \mathrm{Mo}^{1, \mathrm{a}}$, Xiang Zhang ${ }^{2}$,Yibo Zou ${ }^{3}$,Shengmao Shu ${ }^{4}$ \\ ${ }^{1}$ Shcool of Hydropower and Information Engineering, Huazhong University of Science and Technology, Wuhan, Hubei 430074, China \\ ${ }^{2}$ Central China Power Grid Company, Wuhan, Hubei 430077, China
}

\begin{abstract}
The existing electric grid purchase ratio research focuses on the methods of various types of combinations of purchasing and selling business of electricity, and the methods of reasonable risk allocation between different businesses, First, it lacks the consideration of the sensitivity of customer requirements to electricity price changes. Second, it lacks the consideration of optimization of the revenue and risk of hydropower purchase in the provincial electric grid.

Therefore, this paper establishes the multi-objective time-of-use power price model based on customer requirements for time-of-use power price response.It introduces a VaR-based risk assessment method. Also, it proposes a multi-objective optimization model that maximizes the expected revenue on electricity purchase and minimizes the risk of purchasing electricity. The electricity purchase ratio scheme and the electrictiy purchase risk scheme are jointly optimized to obtain the electric grid inter-provincial electrictiy purchase risk decision and the optimal electricity purchase ratio. The results show that the grid company will obtain greater economic benefits while avoiding risks as much as possible after using the power purchase optimization method of this paper.
\end{abstract}

\section{Establishment of time-price model}

\subsection{Customer response curve}

Drawing on the operation mode of the power market in East China, the power sales side and the power purchase side will be divided into peak and low valley sections for operation. The peak time is from 8:00 to 22:00 every day, and the trough time is from 22:00 to 8:00 every day. The peak and valley time-selling electricity price, that is, the peak and the low valley are implemented with different sales price.

According to the principle of consumer psychology, users will have different responses to different electricity prices. Thus changing their power consumption mode, resulting in the effect of peak shaving and valley filling. Therefore, the user's reaction to the electricity price can form a user's reaction curve. The user response curve is studied. After the peak -valley price difference is adjusted multiple times, the electricity price difference and the power difference before and after the adjustment can be counted; then the regression fitting is performed to obtain the response curve of the user in a certain area:

$$
y=f(\mathrm{t}, \mathrm{x})
$$

$\mathrm{Y}$ is the ratio of the electricity consumption after the peak-valley time-sharing electricity price to the electricity consumption and the electricity consumption before the implementation, $x$ is the ratio of the electricity price to the original electricity price after using the peak-to-valley time-of-use price.

In this paper, we use the electricity price comprehensive reaction curve of the literature ${ }^{[2]}$ to fit the formula (2), as shown below:

$\left\{\begin{array}{c}y=0.4319 x^{3}-0.4794 x^{2}-0.4191 x+1.4629(x \leq 1) \\ y=0.329 x^{3}-1.8315 x^{2}-2.5134 x+0.0111(x>1)\end{array}\right.$

According to the peak and valley time division, the corresponding model is established for optimization.

\subsection{Establishment of objective function}

\subsubsection{The first objective function}

Regarding the objective function of time-sharing electricity price ${ }^{[1]}$, there are generally multiple targets with minimum peak load, maximum valley load, and minimum peak-to-valley difference. All of them can achieve the purpose of balancing system load:

(1) Minimize system peak load:

$$
\min _{x_{f}, x_{g}} \max _{t} L=\frac{Q_{t} f\left(t, x_{f}, x_{g}\right)}{\Delta \mathrm{t}}
$$

Maximize system valley load:

$$
\max _{x_{f}, x_{g}} \min _{t} L=\frac{Q_{t} f\left(t, x_{f}, x_{g}\right)}{\Delta \mathrm{t}}
$$

(3) Minimize system peak-to-valley difference: 


$$
\begin{gathered}
\min _{x_{f}, x_{g}}\left[\max _{t} \frac{Q_{t} f\left(t, x_{f}, x_{g}\right)}{\Delta \mathrm{t}}-\min _{t} \frac{Q_{t} f\left(t, x_{f}, x_{g}\right)}{\Delta \mathrm{t}}\right] \\
Q_{t}(t=1,2,3, \ldots, \mathrm{T}) \text { are respectively the electricity }
\end{gathered}
$$
consumption in each period before the implementation of the peak-to-valley time-of-use price, and $\mathrm{T}$ is the total number of time periods, $\Delta \mathrm{t}$ is the time of each time period.

This paper chooses to target the minimum peak-tovalley difference.

\subsubsection{The second objective function}

The satisfaction rate of electricity expenses is taken as the user satisfaction index. The formula is expressed as:

$$
1-\frac{\min _{\mathrm{x}_{f}, \mathrm{x}_{\mathrm{g}}}\left(\mathrm{M}_{1}-\mathrm{M}\right)}{\mathrm{M}}
$$

The $\mathrm{M}$ is the user's electricity purchase fee before using the time-of-use electricity price, $M_{1}$ is the user's electricity purchase fee after using the time-of-use tariff

\subsection{Constraints}

(1) The total number of users is unchanged:

$$
Q_{f}+Q_{g}=Q_{f}^{\prime}+Q_{g}^{\prime}
$$

$Q_{f} 、 Q_{g} 、 Q_{f}^{\prime} 、 Q_{g}^{\prime}$ are respectively the total electricity consumption for each period before and after the implementation of the peak-to-valley time-of-use price..

(2) peak and valley time-of-use electricity price constraints:

Considering the cost of electricity production, the level of social economy, and the affordability of power users, there is a certain limit on the price of electricity:

$$
\left\{\begin{array}{l}
l_{1} p<x_{f}<l_{2} p \\
l_{3} p<x_{g}<l_{4} p
\end{array}\right.
$$

$l_{1} 、 l_{2} 、 l_{3} 、 l_{4}$ are respectively the upper and lower limits of the range of user price changes during peak and valley periods

$$
2 \leq \frac{x_{f}}{x_{g}} \leq 5
$$

$\mathrm{x}_{f} 、 \mathrm{x}_{g}$ are respectively the peak hour electricity price and the valley time electricity price respectively, and $\mathrm{p}$ is the original electricity price of the region.

\subsection{Simplified model:}

$\left\{\begin{array}{c}\min _{x_{f}, x_{g}}\left[\max _{t} \frac{Q_{t} f\left(t, x_{f}, x_{g}\right)}{\Delta \mathrm{t}}-\min _{t} \frac{Q_{t} f\left(t, x_{f}, x_{g}\right)}{\Delta \mathrm{t}}\right] \\ 1-\frac{\min _{\mathrm{x}_{f}, \mathrm{x}_{g}}\left(\mathrm{M}_{1}-\mathrm{M}\right)}{\mathrm{M}}\end{array}\right.$

(10)

$$
\text { s.t }\left\{\begin{array}{c}
1.2 p \leq x_{f} \leq 1.8 p \\
0.3 p \leq x_{g} \leq 0.8 p \\
Q_{f}^{\prime}+Q_{p}^{\prime}+Q_{g}^{\prime}=Q_{f}+Q_{p}+Q_{g} \\
2 \leq \frac{x_{f}}{x_{g}} \leq 5
\end{array}\right.
$$

The NSGA2 algorithm solves the problem with the highest user satisfaction and the minimum peak-to-valley difference, and obtains the optimal peak period electricity price and valley time price.

\section{Introduction of the NSGA-II algorithm}

The NSGA-II algorithm was proposed by Srinivas and Deb on the basis of NSGA in 2000. It is superior to the NSGA algorithm: it uses a fast non-dominated sorting algorithm, and the computational complexity is much lower than that of NSGA; congestion and congestion are adopted. The comparison operator replaces the shared radius shareQ that needs to be specified, and serves as the winning criterion in the peer comparison after quick sorting, so that individuals in the quasi-Pareto domain can be extended to the entire Pareto domain and evenly distributed, maintaining the Diversity of population .It introduces an elite strategy, expands the sampling space, prevents the loss of the best individual, and improves the computational speed and robustness of the algorithm.

NSGA-II is based on the first generation of nondominated sorting genetic algorithm, and its improvement is mainly for the three aspects mentioned above:

(1)A fast non-dominated sorting algorithm is proposed, which reduces the computational complexity on the one hand, and merges the parental population with the progeny population on the other hand, so that the next generation of populations are selected from double space, thus retaining The best of all individuals;

(2) Introduce an elite strategy to ensure that some excellent individual individuals will not be discarded during the evolution process, thus improving the accuracy of the optimization results;

(3) The use of congestion and congestion comparison operators not only overcomes the deficiencies in NSGA that require artificially assigned parameters, but also serves as a comparison criterion between individuals in the population, so that individuals in the quasi-Pareto domain can be uniformly extended to the entire Pareto. The domain ensures the diversity of the population.

\section{Multi-objective optimization model of benifit-risk ${ }^{[3]}$ of electricity purchasing ratio}

The goal of grid company risk management is to eliminate the volatility of its profits, and managers must be able to assess potential losses in grid operations. The power purchase risk of the grid company refers to the possible adverse changes in the value of the assets due to changes in the price of the electricity market and changes in the volatility of the price of electricity. The risk is generally 
measured in the form of price volatility, which will cause changes in the grid company's rate of return.

This paper starts with the application of portfolio theory in the risk analysis of purchasing power, assuming that there is no price fluctuation in the contract market of peak and valley segments, and regards the cost of purchasing electricity in the peak and valley segments of the futures market as a portfolio and using VAR value to analysis its fluctuations in electricity purchase costs.

\subsection{Calculation of portfolio $\operatorname{VaR}^{[4]}$}

VaR refers to the worst expected loss of a certain portfolio under certain confidence levels in the future, under the normal fluctuation of the market, which can be expressed as $P(\Delta V \leq V)=1-c$, In this formula: $\Delta V$ is the change in the value of the portfolio when the market changes unfavorably: $\mathrm{c}$ is the confidence level: $\mathrm{V}$ is the value at risk under the confidence level $\mathrm{c}$. The maximum loss measured by $\Delta V$ is equal to the VaR value of the portfolio at a given confidence level c.

We take the purchase cost of the spot market peak period as the first investment, and the purchase cost of the spot market during the valley period as the second investment ${ }^{[5]}$, assuming that $\beta_{1} 、 \beta_{2}$ are respectively the ratio of the first investment and the second investment purchase to the total purchase price of the futures market. The average electricity price of the first investment is $p_{f x}$, the fluctuation standard deviation is $\sigma_{1}$; the average electricity price of the second investment is $p_{g x}$; the fluctuation standard deviation is $\sigma_{2}$; the total electricity purchase is $\mathrm{Q}_{\mathrm{x}}$;

The total investment cost is:

$$
\mathrm{G}_{\mathrm{x}}=\left(\beta_{1} p_{f x}+\beta_{2} p_{g x}\right) \mathrm{Q}_{\mathrm{x}}
$$

The variance of investment fluctuations is:

$\sigma^{2}=\beta_{1}{ }^{2} \sigma_{1}^{2}+\beta_{2}{ }^{2} \sigma_{2}^{2}+2 \beta_{1} \beta_{2} \sigma_{1} \sigma_{2} p$

$p$ is the correlation coefficient of the electricity price in the peak and valley period.

The value of risk is:

$$
\text { Var }=\alpha S_{1} \sqrt{T} \sqrt{\beta_{1}^{2} \sigma_{1}^{2}+\beta_{2}^{2} \sigma_{2}^{2}+2 \beta_{1} \beta_{2} \sigma_{1} \sigma_{2} p}
$$

\subsection{Establishment of the income maximization model}

The purchase side is divided into the same peak-to-valley period, corresponding to different price levels; from the provincial, provincial monthly contract market, spot market power purchase, analysis the daily purchase of electricity, using mathematical functions expressed as follows:

$$
\max R=\max \{E(S-G)\}
$$

$\mathrm{S}$ is the income of electricity sales, $\mathrm{G}$ is the cost of electricity purchase, $\mathrm{R}$ is the expected return on electricity purchase; $\mathrm{E}$ is the mean operator;

(1) Electricity sales revenue: In the case of implementing peak-to-valley time-of-use tariffs on the electricity-selling side, the sales revenue is related to the peak-to-valley time-of-use tariff plan and the corresponding load response. The sales revenue $\mathrm{S}$ is:

$$
\mathrm{S}=x_{f} \mathrm{Q}_{f}+x_{g} \mathrm{Q}_{g}
$$

$\mathrm{Q}_{f}$ is the load demand for the peak period; $\mathrm{Q}_{\mathrm{g}}$ is the load demand for the valley period; $\mathrm{x}_{\mathrm{f}}$ is the electricity price during the peak period of the electricity sales side; $\mathrm{x}_{\mathrm{g}}$ is the electricity price during the valley period of the electricity sales side

(2) Cost of electricity purchase: Calculate the cost of electricity purchase, the cost of electricity purchase $\mathrm{G}$ is: $\mathrm{G}=\left(1-\lambda_{1}\right) \mathrm{Q}_{f} p_{f h}+\lambda_{1} \mathrm{Q}_{f} p_{f x}+\left(1-\lambda_{2}\right) \mathrm{Q}_{g} p_{g h}+\lambda_{2} \mathrm{Q}_{g} p_{g x} ;$

$\lambda_{1}, \lambda_{2}$ are respectively the ratio of the purchased electricity in the spot market to the total purchased electricity in the peak and valley periods respectively; $p_{f h}$, $p_{f x}$ are respectively the peak-time contract market and the spot market hydropower unit purchase cost; $p_{g h}, p_{g x}$ are respectively the electricity purchase expenses for hydropower in the valley period contract market and the spot market.

\subsection{Simplified model:}

Establishing the multi-objective optimization model of benifit-risk of electricity purchasing ratio ,solving the expected value of electricity purchase, the risk of purchasing electricity, purchasing ratio of peaks and valleys in the provinces and provinces.

Objective function:

$\left\{\begin{array}{c}\max R=\max \{E(S-G)\} \\ \min \mathrm{VaR}=\alpha \sqrt{\mathrm{T}} \sqrt{\beta_{1}{ }^{2} \sigma_{1}{ }^{2}+\beta_{2}{ }^{2} \sigma_{2}{ }^{2}+2 \beta_{1} \beta_{2} \sigma_{1} \sigma_{2} p}\end{array}\right.$

Constraints: Electricity balance, purchase power constraints.

(1)Electricity balance

$$
\sum_{i=1}^{4}\left(Q_{i} / Q\right)=1
$$

$Q_{1}, Q_{2}$ are respectively the electricity of Contract market in peak and valley time, $Q_{1}, Q_{2}$ are respectively the electricity of spot market in peak and valley time, $Q$ is the total load demand:

(2) purchase power constraints

$X_{i}, \min \leq X_{i} / Q \leq X_{i}, \max (i=1,2, \ldots, 4$,

$X \mathrm{i}, \max 、 \mathrm{Xi}, \min$ is the largest and smallest ratio of $\mathrm{Xi}$ accounts for the total load demand;

\subsection{Solve}

Solve the corresponding power purchase ratio scheme and its benefits and risks through NSGA-II algorithm; obtain the power purchase ratio scheme under different expected benefits for decision makers with different risk preferences. 


\section{Examples analysis}

4.1 This example uses a set of summer typical load data in a certain area for simulation calculation. The specific data is shown in Table 1.

Table 1.A set of summer typical load data in an instance

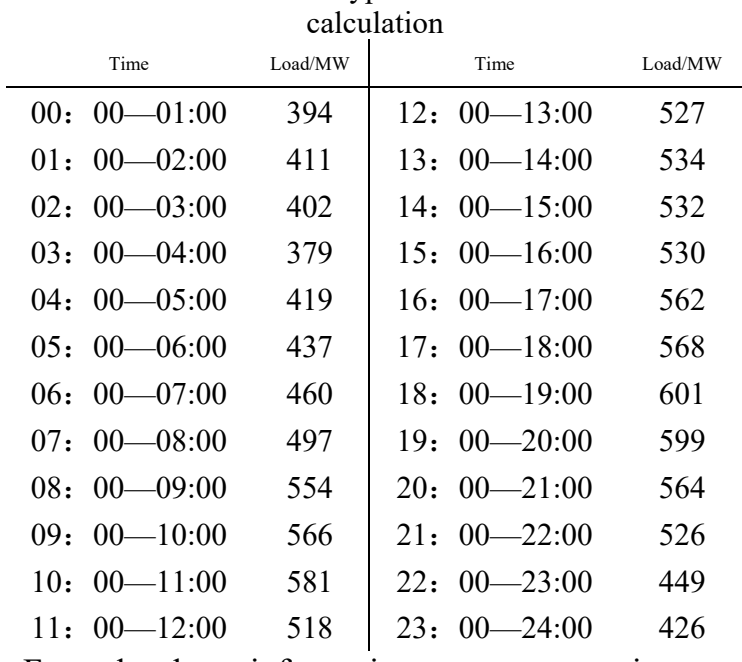

From the above information, we can recognize:

The maximum load is $601 \mathrm{MW}$, the minimum load is $379 \mathrm{MW}$, and the peak-to-valley difference is $222 \mathrm{MW}$.

4.2 After studying the user response curve and adjusting the peak-to-valley price difference multiple times, the electricity price difference and the power difference before and after the adjustment can be counted; then the regression fitting is performed to obtain the response curve of the user in the area

$$
\left\{\begin{array}{l}
y=0.4319 x^{3}-0.4794 x^{2}-0.4191 x+1.4629(x \leq 1) \\
y=0.329 x 3-1.8315 x 2+2.5134 x-0.0111(x>1)
\end{array}\right.
$$

Before the implementation of the time-of-use tariff, the user purchase price is $70.147 \$ / \mathrm{MWh}$. Based on the electricity market time division in East China, the purchase and sale of electricity are divided into peak time and valley time: The peak hours are from 8:00 to 22:00 (excluding 22:00) and the trough is from 22:00 to 8:00 (excluding 8:00). According to the economic development level, user affordability and power cost analysis of the place, the local price control department stipulates the user peak and valley time price:

$$
\left\{\begin{array}{l}
1.2 p \leq x_{f} \leq 1.8 p \\
0.3 p \leq x_{\mathrm{g}} \leq 0.8 p
\end{array}\right.
$$

The goal is to minimize the peak-to-valley difference and the highest user satisfaction in the system:

$$
\left\{\begin{array}{c}
\min _{x_{f}, x_{g}}\left[\max _{t} \frac{Q_{t} f\left(t, x_{f}, x_{g}\right)}{\Delta \mathrm{t}}-\min _{t} \frac{Q_{t} f\left(t, x_{f}, x_{g}\right)}{\Delta \mathrm{t}}\right] \\
\max _{x_{\mathrm{f}}, x_{g}}\left[1-\frac{\mathrm{M}_{1}-\mathrm{M}_{0}}{\mathrm{M}_{0}}\right]
\end{array}\right.
$$

The optimal peak-time electricity price is $87.76 \$ / \mathrm{MWh}$ and the valley-time price is $43.53 \$ / \mathrm{MWh}$ by NSGA-II algorithm. After using the time-sharing electricity price, the system peak-to-valley difference is $135.7979 \mathrm{MWh}$, the satisfaction is $100 \%$, and the peak-to-valley timesharing is used. The load comparison before and after the electricity price is shown in Figure 2.

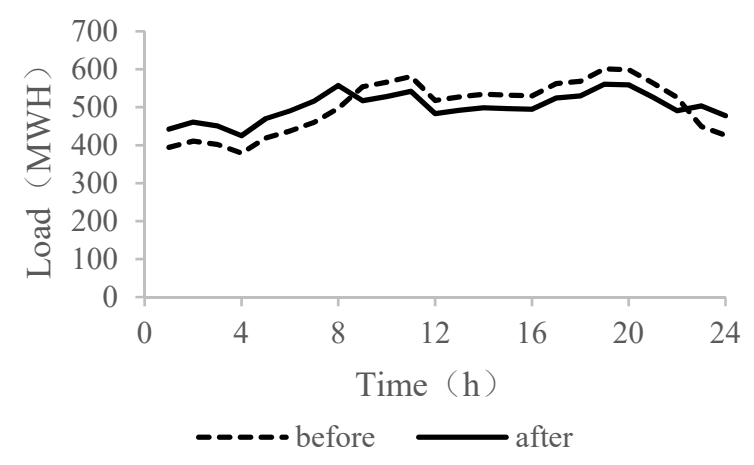

Figure 2. Load comparison chart before and after using peak and valley time-of-use electricity price

4.3 According to the trial operation data of East China Power Market, the average electricity price distribution obeys the normal distribution. The peak market price is $45 \$ / \mathrm{MWh}$, the real-time electricity price is $36.925 \$ / \mathrm{MWh}$, the standard deviation is $3.693 \$ / \mathrm{MWh}$; the market price of the valley contract is $33.35 \$ / \mathrm{MW}$, real-time electricity price average $30.441 \$ / \mathrm{MWh}$, standard deviation $2.52 \$$ / MWh.

Using the NSGA-II algorithm to calculate the expected revenue maximization model and the risk minimization model, the corresponding purchase ratio scheme and its benefits and risks are obtained; The power purchase ratio scheme is chosen by decision makers with different risk preferences. The data is shown in Table 2 .

Table 2. The power purchase ratio scheme derived from the calculation of the example and its benefits and risks

\begin{tabular}{|c|c|c|c|}
\hline $\begin{array}{c}\text { Expected } \\
\text { revenue(\$/MWh) }\end{array}$ & $\begin{array}{c}\text { Conditional } \\
\text { value-at- } \\
\text { risk(\$/MWh) }\end{array}$ & $\begin{array}{c}\text { Peak time } \\
\text { spot } \\
\text { market } \\
\text { purchase } \\
\text { ratio }\end{array}$ & $\begin{array}{c}\text { Low-spot } \\
\text { period } \\
\text { spot } \\
\text { market } \\
\text { purchase } \\
\text { ratio }\end{array}$ \\
\hline 358504.5935 & 0.0000 & 0.0000 & 0.0000 \\
\hline 359284.1653 & 690.9455 & 0.0095 & 0.0162 \\
\hline 361253.0561 & 2130.0935 & 0.0437 & 0.0139 \\
\hline 362634.9691 & 3140.5231 & 0.0689 & 0.0071 \\
\hline 365654.3750 & 5948.8991 & 0.0982 & 0.1008 \\
\hline 367323.4530 & 6712.4182 & 0.1467 & 0.0169 \\
\hline 369070.0225 & 8029.2284 & 0.1766 & 0.0169 \\
\hline 371006.6208 & 9475.4805 & 0.2107 & 0.0129 \\
\hline 373383.6626 & 11276.6783 & 0.2508 & 0.0152 \\
\hline 375141.7690 & 12713.0635 & 0.2738 & 0.0445 \\
\hline 376410.7758 & 13668.9064 & 0.2955 & 0.0445 \\
\hline 377660.5300 & 14865.5099 & 0.3035 & 0.1008 \\
\hline 379616.6069 & 15931.3106 & 0.3610 & 0.0001 \\
\hline 382335.9360 & 18311.8240 & 0.3866 & 0.0873 \\
\hline 383686.1922 & 19388.1260 & 0.4065 & 0.1008 \\
\hline 385522.2590 & 20424.9240 & 0.4590 & 0.0121 \\
\hline & & & \\
\hline
\end{tabular}




\begin{tabular}{|l|l|l|l|}
\hline 386993.0422 & 21534.7136 & 0.4842 & 0.0121 \\
\hline 389172.4251 & 23141.9502 & 0.5243 & 0.0000 \\
\hline 391343.8165 & 24787.2703 & 0.5609 & 0.0023 \\
\hline 391913.0398 & 25216.8055 & 0.5707 & 0.0023 \\
\hline 395518.6818 & 28047.1179 & 0.6242 & 0.0363 \\
\hline 397856.4302 & 29712.7636 & 0.6714 & 0.0059 \\
\hline 398839.8480 & 30465.1444 & 0.6874 & 0.0093 \\
\hline 399782.5789 & 31411.7750 & 0.6873 & 0.0776 \\
\hline 400439.1223 & 32092.7704 & 0.6874 & 0.1240 \\
\hline 402444.0490 & 33256.4297 & 0.7437 & 0.0317 \\
\hline 403819.2763 & 34337.0239 & 0.7642 & 0.0445 \\
\hline 405086.3844 & 35292.9212 & 0.7858 & 0.0445 \\
\hline 405935.4366 & 36976.0288 & 0.7437 & 0.2821 \\
\hline 408811.9063 & 38209.7074 & 0.8423 & 0.0748 \\
\hline 408938.3306 & 38309.9796 & 0.8441 & 0.0762 \\
\hline 410781.1312 & 39640.3066 & 0.8796 & 0.0595 \\
\hline 413137.3313 & 41276.8504 & 0.9301 & 0.0167 \\
\hline 415205.6915 & 42802.3222 & 0.9682 & 0.0052 \\
\hline 417306.0496 & 44458.0003 & 0.9987 & 0.0280 \\
\hline 418011.1425 & 45141.6218 & 1.0000 & 0.0731 \\
\hline 418382.7519 & 45942.5705 & 0.9751 & 0.2043 \\
\hline 420477.7690 & 47749.5535 & 0.9987 & 0.2555 \\
\hline 422942.6438 & 51719.9812 & 0.9502 & 0.6358 \\
\hline 426162.6466 & 54240.9796 & 1.0000 & 0.6577 \\
\hline 426268.5825 & 54416.4312 & 0.9982 & 0.6728 \\
\hline 427569.0740 & 55978.4817 & 0.9987 & 0.7642 \\
\hline 428627.0025 & 57948.5460 & 0.9761 & 0.9346 \\
\hline 428989.2576 & 58278.2207 & 0.9802 & 0.9436 \\
\hline 430934.8416 & 60109.0296 & 1.0000 & 1.0000 \\
\hline
\end{tabular}

This work was supported by the National Key R\&D Program of China (No. 2016YFC0402210) and the National Natural Science Foundation of China (No. 51479075), the Fundamental Research Funds for the Central Universities ( No. 2017KFYXJJ199) and the Science and Technology Project of State Grid Corporation (No. SGHZ0000DKJS1800195).

\section{Reference}

1. Guanqi Liu, Jian Zhang, Wei Liu. Research on Tou Pricing based on constomer response curve [J].Journal of North China Electric Power University,2005(03):23-27.

2. Lin Gao.The Application Study of time-of-use Decision Price Optimization Model Based on Customer's Response [D]. Hefei University of Technology, 2010.

3. Qing WANG, Guo CHEN, Min LIU. A New Risk Decision-making Theory : Based on Value and Risk[J].China Management Science, 2014,22(03):42-50.

4. Yu Shan. Calculation Methods of the Value at Risk [D]. Chongqing University, 2013.

5. Wei Chen. Financial Risk Management for Grid corporation in power market [D]. Southeast University, 2005.

\section{Conclusion}

In this paper, based on the sensitivity of users to electricity price changes, considering the goal of minimizing system peak-to-valley load and maximizing user satisfaction, a multi-objective time-of-use electricity price model based on user response is established, which improves the feasibility of time-sharing electricity price scheme. It laid the foundation for establishing the largest expected model of power purchase. In the establishment of the largest model of expected revenue from electricity purchases, the inter-provincial contract and the electricity purchase ratio of the spot market were considered, and the conditional risk value based on the portfolio theory was introduced as the risk index, and the expected return benefit of the purchase of electricity and the risk of purchasing electricity were proposed . The minimized multi-objective optimization model realizes the joint optimization of the electricity purchase ratio scheme and the power purchase risk scheme, and coordinates the economic benefits of the user and the grid.

\section{Acknowledgement}

